\title{
Norois
}

Environnement, aménagement, société

$247 \mid 2018$

Recherche touristique : perspectives latinoaméricaines

\section{Développement touristique et appropriation foncière : le rôle des « mafias agraires » à Tulum au Mexique}

Tourism development and land appropriation : the role of agrarian mafias in

Tulum, Mexico

Desarrollo turístico y apropiación de la tierra: el papel de las "mafias agrarias" en Tulúm, México

\section{Gustavo Marín Guardado}

\section{CpenEdition}

Journals

Édition électronique

URL : http://journals.openedition.org/norois/6508

DOI : $10.4000 /$ norois. 6508

ISBN : 978-2-7535-7629-2

ISSN : 1760-8546

Éditeur

Presses universitaires de Rennes

Édition imprimée

Date de publication : 19 septembre 2018

Pagination : 31-47

ISBN : 978-2-7535-7570-7

ISSN : 0029-182X

Référence électronique

Gustavo Marín Guardado, « Développement touristique et appropriation foncière : le rôle des « mafias agraires » à Tulum au Mexique », Norois [En ligne], 247 | 2018, mis en ligne le 19 septembre 2020, consulté le 05 janvier 2021. URL : http://journals.openedition.org/norois/6508 ; DOI : https://doi.org/ 10.4000/norois. 6508 


\section{INTRODUCTION}

Cette contribution examine les liens entre le développement du tourisme et les logiques d'appropriation territoriale à travers lesquelles certains groupes d'intérêt politique et économique parviennent à s'imposer sur d'autres dans un contexte de forte spéculation immobilière, d'incertitude juridique et de confrontation sociale associée à des mécanismes institutionnels de dépossession foncière. J'exposerai plus spécifiquement le cas de l'ejido ${ }^{1}$ José Maria Pino Suarez situé dans la municipalité de Tulum, au Mexique, pour montrer l'incertitude entourant la propriété du sol dans cette localité, mais aussi les conditions de vulnérabilité et d'extrême difficulté pour les populations locales qui tentent de prendre part à l'économie touristique. Tant sur le marché immobilier que dans le cadre de projets de développement, cette vulnérabilité tient notamment à l'existence d'une élite et d'une structure du pouvoir constituant une forme locale de «mafia agraire » et s'imposant par le biais d'un processus d'appropriation de l'espace particulièrement agressif.

Le contexte étudié est marqué par des processus historiques liés à la réorganisation des groupes ethniques, l'exploitation forestière, l'économie domestique rurale et les politiques de redistribution agraire mises en œuvre par l'État mexicain au $\mathrm{Xx}^{\mathrm{e}}$ siècle. Dans cette configuration, les phénomènes de valorisation foncière et de spéculation immobilière ainsi que les luttes pour le territoire ont acquis une importance fondamentale avec l'apparition du tourisme (Marín Guardado, à paraître). Des dynamiques comme celles signalées plus tôt ont ainsi émergé sous l'action des groupes dominants soucieux de faciliter les investissements dans des projets de développement touristique. Ces projets impliquent l'éviction systématique des groupes locaux, sur le plan spatial mais aussi comme bénéficiaires potentiels d'un tel développement. L'ejido José Maria Pino Suarez et la localité de Tulum sont donc au centre d'un processus historique de construction d'un lieu

1. L'ejido est le régime foncier sous lequel s'effectue l'essentiel de la redistribution agraire mise en œuvre par l'État mexicain au $\mathrm{xx}^{\mathrm{e}}$ siècle. Cet héritage de la Révolution mexicaine instaure un système de propriété collective de la terre et constitue légalement la communauté agraire formée par l'ensemble des ejidatarios, c'est-à-dire des paysans bénéficiant d'un droit d'usufruit inaliénable, transmissible de génération en génération. Les terres ainsi distribuées par le ministère de la Réforme agraire sont administrées collectivement par une assemblée agraire composée de l'ensemble des ejidatarios, ainsi que par leurs représentants élus au sein d'un commissariat ejidal. touristique caractérisé par le rôle des capitaux internationaux et des logiques globalisées. Articulés à des structures économiques et de pouvoir à l'échelle nationale et locale, ces processus constituent un mécanisme fondamental en termes d'appropriation de l'espace et du point de vue de l'accumulation du capital (López et Marín, 2012 ; López, 2010 ; Marín, 2010, 2015). Il s'agit d'un processus de grande ampleur qui, selon différentes modalités et comme dans d'autres secteurs de l'économie mondiale, se caractérise par le recours à la fraude, à la spéculation et à la dépossession (Harvey, 2003).

Pour éclairer ce phénomène, la réflexion s'appuiera sur un travail ethnographique mené à Tulum entre 2006 et 2016. Le dispositif d'enquête repose notamment sur la réalisation de 68 entretiens approfondis avec différents acteurs impliqués dans les conflits pour le contrôle du foncier touristique (ejidatarios, petits propriétaires, entrepreneurs touristiques et intermédiaires). Parallèlement au recueil de ces différents récits, lequel permet d'appréhender la diversité des points de vue et des intérêts engagés dans ces luttes locales, l'analyse se fonde sur un corpus d'articles de presse locale, nationale et internationale traitant de ces conflits ainsi que sur la consultation d'archives agraires issues, notamment, du Registre Agraire National (RAN). L'article commencera par expliciter le concept de «mafia agraire » en montrant son caractère opératoire pour penser les logiques foncières inhérentes au développement touristique dans cette partie de la péninsule du Yucatan. Après avoir caractérisé plus précisément les pratiques et logiques d'acteurs que ce terme recouvre, je retracerai le processus de mise en tourisme à l'échelle locale et régionale ainsi que ses effets sur l'urbanisation de Tulum. Passée cette mise en contexte, l'article exposera la genèse des conflits depuis la mise en place de la structure foncière locale et les différentes phases de ce processus conflictuel jusqu’à la période actuelle.

\section{ESPACES DISPUTÉS, « MAFIAS AGRAIRES » : RETOUR SUR UN PROCESSUS DE « DÉPOSSESSION INTÉGRALEMENT PLANIFIÉE »}

L'histoire du tourisme au Mexique tend à occulter le processus d'appropriation territoriale inhérent au développement du phénomène touristique. À l'heure 
actuelle, la construction de centres touristiques ayant impliqué la dépossession de terres appartenant à des paysans, des pêcheurs ou des petits propriétaires, principalement par le biais d'expropriations précédant leur revente à des investisseurs privés, reste relativement peu étudiée (Marín, 2015). Un tel phénomène s'observe notamment dans le cas des «Centres Intégralement Planifiés (CIP) » développés à partir des années 1960. Dans plusieurs cas, ces expropriations ont été accompagnées du déplacement forcé de petits villages côtiers pour permettre la construction de complexes hôteliers destinés aux élites du monde entier. Aussi, il a été montré que le développement du tourisme est systématiquement lié à des phénomènes de spéculation immobilière impliquant des institutions étatiques, des consortia entrepreneuriaux, des élus, des intermédiaires, des caciques locaux et des aménageurs qui se sont progressivement approprié de grandes quantités de terres situées à l'intérieur du pays ou sur les littoraux. Souvent, ce phénomène implique une utilisation factieuse des institutions de l'État et le recours légal à l'expropriation. Dans d'autres cas, cela passe par la persuasion économique de localités pauvres ou, simplement, par l'intimidation et la violence. Si un tel phénomène s'avère systématique, il se traduit également par d'innombrables cas de négociations et de résistances.

Le développement touristique de la péninsule du Yucatan, incluant les villes de Mérida, Campeche et Chetumal, les îles de Cozumel, Isla Mujeres et Holbox, mais aussi le centre touristique de Cancun, la Riviera Maya et la Costa Maya, a donné lieu à de nombreux projets touristiques et urbains marqués par ce type de rapports de pouvoir. L'un des traits saillants de la plupart des projets est l'importance des stratégies de spéculation foncière, un phénomène d'autant plus complexe qu'il implique souvent la participation de ce qui apparaît comme une «mafia agraire ».

Le terme de «mafia agraire » est communément utilisé par les journalistes de la péninsule du Yucatan pour qualifier des groupes d'intérêt entrepreneuriaux essayant de s'approprier des terres à des fins spéculatives avec la complicité de fonctionnaires gouvernementaux et de représentants d'institutions fédérales. Dans cette recherche, il m’a semblé pertinent d'adopter ce terme mais aussi de préciser sa portée conceptuelle pour en faire un outil descrip- tif et analytique. En ce sens, j'entends par « mafia agraire » un réseau complexe de personnes et d'institutions composé d'entrepreneurs, d'élus, de fonctionnaires gouvernementaux, d'intermédiaires et de courtiers en immobilier. Ces acteurs disposent de grandes quantités de capitaux, mais également de conseillers, d'avocats, de notaires, d'opérateurs, de prête-noms et de relations dans différents milieux allant des hautes sphères politiques aux autorités agraires locales. Ces différentes ressources leur permettent de mettre en œuvre des procédures officielles auprès de l'administration agraire et du cadastre, de bénéficier de recours en justice ou d'expertises afin d'obtenir des terres en propriété collective, à faible prix et par n'importe quel moyen légal ou extralégal, pour ensuite effectuer des transactions à haut rendement dans le secteur immobilier. Ces spéculateurs constituent un réseau de relations particulièrement dense à l'échelle de la péninsule du Yucatan. Ils interviennent dans une multitude de processus de parcellement et d'appropriation de terrains communaux à la valeur marchande croissante. Malgré l'ampleur de ce réseau, celui-ci s'articule autour d'une poignée de figures dominantes comprenant des politiciens notoires et des entrepreneurs yucatèques, d'anciens gouverneurs, un ancien banquier ainsi qu'un certain nombre de parents et d'amis de cette pléiade d'accapareurs et de spéculateurs. Ces derniers bénéficient en outre de relais au sein d'institutions clés comme le Registre Agraire National, le Registre Publique de la Propriété, les tribunaux agraires, principalement dans les états du Yucatan et du Quintana Roo.

Les stratégies d'appropriation territoriale déployées par ces groupes sont multiples. L'une des plus courantes repose sur la création de sociétés spécialisées dans l'aide juridique au parcellement, à la titrisation et la vente de terres d'usage commun ainsi que dans l'urbanisation de terrains et le développement de projets immobiliers. Une telle stratégie permet en effet un contact direct avec de nombreux ejidos de la péninsule et un accès privilégié à l'information permettant d'intervenir au sein des assemblées agraires, de prendre part aux négociations et aux procédures officielles. Cette position permet également de manipuler les décisions des ejidatarios avec la complicité de leaders agraires corrompus, ou de contourner la loi. Partant, ces derniers développent des stratégies agressives visant l'appropriation de 
terres ejidales à grande échelle dans des espaces revalorisés par la croissance urbaine et le développement touristique. Un tel phénomène s'observe notamment dans les environs de Mérida et au sein des ejidos de Kanasín et Chablekal, dans le Yucatán ou, par exemple, dans les ejidos de Holbox, Chiquilá, Cozumel, Playa del Carmen, Puerto Morelos et Tulum dans l'état du Quintana Roo.

\section{Tulum, NOUVEau Pôle DE DÉVELOPPEMENT TOURISTIQUE}

La ville de Tulum se situe au centre de la façade littorale du Quintana Roo, à l'extrémité sud de la Riviera Maya. Port commercial à l'époque préhispanique, la cité maya comprenait également un observatoire astronomique dont les vestiges archéologiques constituent aujourd'hui l'un des principaux attraits pour le tourisme international. À la fin du XIX ${ }^{e}$ siècle et au début du $\mathrm{xx}^{\mathrm{e}}$, Tulum devint un centre cérémoniel pour les insurgés mayas durant la Guerre des Castes (1847-1901). À l'issue de la guerre, la localité fit l'objet d'une restructuration agraire. Entre les années 1930 et les années 1980, l'État mexicain mit en ouvre la réforme agraire et la distribution de terres ejidales dans le cadre d'une stratégie de colonisation du Quintana Roo. Cette redistribution s'effectue alors au profit de communautés mayas issues de l'ensemble de la péninsule ou de paysans originaires de différentes régions du pays.

Pendant des décennies, l'activité économique locale s'est organisée autour de la production de résine de latex (chicle) destinée au marché mondial, de l'agriculture de subsistance et de la pêche, associées à d'autres activités de la vie rurale. Dans ce contexte, le développement de Tulum resta limité et la population locale ne dépassait pas 2000 habitants en 1990 (Juárez, 2002; Marín, 2010). Si la présence de touristes venant visiter le site archéologique et le village remonte aux années 1930, celle-ci se limite à des groupes réduits se déplaçant dans de petits avions depuis l'île de Cozumel. S'agissant principalement d'excursions à la journée, cette activité ne génère cependant pas de retombées économiques significatives pour la population locale. Pour autant, la situation commença à changer après la création de l'un des principaux centres touristiques du monde à Cancun, au milieu des années 1970. Cette évolution est traduite par la construction d'une route côtière et, plus généralement, par une amélioration des moyens de transport et de nouvelles possibilités d'emplois pour les populations locales. La fréquentation touristique connaît dès lors une croissance ininterrompue liée à la présence d'un site archéologique avec vue sur la mer, de plages paradisiaques et d'une atmosphère mystique, loin de la vie urbaine.

Le déclin de la production de chicle, la crise agricole et l'influence de Cancun ont ouvert la voie à un développement touristique rapide à Tulum. Dans un premier temps, la localité devient une destination pour le tourisme alternatif. L'activité se concentre autour d'un ensemble de cabanes situées en bord de plage à proximité des vestiges archéologiques, sans luxe ni commodités hormis la possibilité de jouir de l'isolement, de la nature et de la culture locale. À cette époque, le village appartient à la municipalité de Cozumel. À partir de 1993, Tulum relève de la municipalité de Solidaridad nouvellement constituée dans la partie continentale de l'ancienne municipalité. Ce changement s’inscrit plus généralement dans la nouvelle dynamique du développement touristique et participe de la construction de la région touristique aujourd'hui connue sous le nom de Riviera Maya (Marín, 2010). De cette manière, les ejidos de Tulum deviennent un élément majeur du nouveau projet de développement touristique.

Cette restructuration liée aux nouvelles dynamiques touristiques locales, traduite par l'arrivée de nouvelles personnes à la recherche d'un lieu de vie ou d'un endroit pour débuter une activité commerciale, a contribué à la croissance rapide de la ville. Ainsi, le développement de la vente de terres, l'apparition de nouveaux foyers de peuplement sur la plage, l'abandon des activités agricoles, la croissance démographique et urbaine alimentée par de nombreux migrants, la présence de milliers de touristes résidentiels et de nouveaux acteurs ont progressivement donné vie à une société plus complexe du point de vue de son économie et de sa structure sociale (Juárez, 2002; Marín, 2010). Une société en partie constituée par des populations mayas autochtones ou issues d'autres parties de la péninsule, mais aussi par de nouveaux résidents en provenance du Mexique et d'autres parties du monde, dessinant un lieu divers et cosmopolite.

En 2008, Tulum accède à son tour au rang de municipalité. La nouvelle entité municipale est dotée d'un territoire de $2090,43 \mathrm{~km}^{2}$ appartenant 
jusque-là à la municipalité de Solidaridad. Le territoire concerné (figure 1 - planche III) intègre la ville de Tulum, constituée en chef-lieu, et d'autres localités de plus petite taille. L'ensemble concerné représentait en 2015 une population de 32700 habitants, dont plus de 21000 regroupés au chef-lieu (INEGI, 2015) ${ }^{2}$.

La municipalité de Tulum intègre également 45 kilomètres de plages paradisiaques parallèles à la barrière de corail la plus importante au monde après celle d'Australie (figures 2-3 - planche IV), ainsi que de vastes espaces de mangrove et de forêt dotés d'une importante biodiversité. Le territoire municipal compte par ailleurs avec de nombreux cenotes, ces cavités naturelles liées à la nature calcaire du sol dans la péninsule du Yucatan et mettant à jour un système de rivières souterraines au rôle écologique majeur, considéré comme l'un des plus importants au monde.

En 2016, les principales destinations du Quintana Roo - principalement Cancun et la Riviera Maya disposaient d'une infrastructure touristique dépassant 97600 chambres d'hôtel et affichaient une fréquentation supérieure à 11 millions de touristes. La même année, les retombées économiques du tourisme s'élevaient à plus de 8,6 milliards de dollars soit 38,5\% des revenus touristiques nationaux (SEDETUR, 2016)³. La Riviera maya - plus spécifiquement constituée par les municipalités de Solidaridad et Tulum - affichait quant à elle une capacité d'accueil de plus de 45000 chambres d'hôtels et une affluence touristique de 4,8 millions de visiteurs générant plus de 3 milliards de dollars de recettes annuelles. Dans ce contexte, la municipalité de Tulum comprenait 144 hôtels pour un total de 7082 chambres. Si l'on ne dispose pas à ce jour de données officielles précises à l'échelle municipale, ces chiffres permettent de mesurer le poids des retombées du tourisme en termes de création de richesses, à l'échelle locale et régionale (tableau 1). L'importance de cette manne financière et les perspectives de développement prometteuses pour l'industrie touristique qu'elle suscite ont engendré une situation complexe à Tulum, ainsi qu'une conflictualité permanente pour le territoire et l'accès aux ressources. C'est dans ce contexte que les populations locales, et plus particulièrement les ejidatarios, tentent de protéger et d'administrer leurs biens tandis que de nombreux entrepreneurs font état de projets d'investissements massifs. Dans cette configuration, les pouvoirs publics font figure d'« intermédiaires du progrès ${ }^{4}{ }$ et d'administrateurs de paradis.

\section{LA STRUCTURE AgRAIRE LOCALE}

L'histoire de la ville de Tulum est étroitement liée à la formation et au développement de trois ejidos : l'ejido Tulum, fondé en 1935 par des populations mayas natives de la localité; le Nouveau Centre de Population Ejidale (NCPE) $)^{5}$ José Maria Pino Suarez, créé en 1973 par un groupe de paysans originaires

\begin{tabular}{|l|c|c|c|}
\hline Indicateur & Quintana Roo & Riviera Maya & Tulum \\
\hline Nombre de chambres d'hôtels & 97606 & 45217 & 7082 \\
\hline Nombre de touriste & 11186645 & 4790056 & $900000^{*}$ \\
\hline Retombées économiques (en millions de dollars) & 8602,48 & 3017,74 & 472,53 \\
\hline
\end{tabular}

*Estimation à partir des statistiques officielles pour la Riviera Maya, proportionnellement au nombre de chambres d'hôtels.

Tableau 1 : Indicateurs touristiques pour le Quintana Roo, la Riviera Maya et la municipalité de Tulum en 2016 (Source : SEDETUR, 2016) Tourist indicators for Quintana Roo, Riviera Maya and Tulum municipality in 2016

Indicadores turísticos para Quintana Roo, la Riviera Maya y el municipio de Tulúm en 2016

2. Lors du Recensement de 2010, la municipalité de Tulum enregistrait une population légèrement supérieure à 28000 habitants dont 18000 pour la seule ville de Tulum. Plus récemment, dans le cadre de l'enquête intercensitaire effectuée en 2015, la municipalité affichait 32700 habitants mais ne fournissait pas de données pour les localités inférieures à 50000 habitants. Pour cette raison, nous nous basons sur une estimation de croissance proportionnelle à la croissance moyenne de la municipalité, laquelle nous conduit à estimer à environ 21000 habitants la population actuelle de la ville de Tulum (INEGI, 2015).

3. En 2016, le Mexique affiche un record historique avec 35 millions de visiteurs internationaux et une captation de devises touristiques atteignant 19,5 milliards de dollars (SECTUR, 2017).

\footnotetext{
4. Ce terme est emprunté à Fernando Salmerón (1998) qui l'a notamment utilisé pour analyser les liens entre des entrepreneurs et des réseaux de pouvoir afin de saisir le rôle de fonctionnaires gouvernementaux, d'investisseurs et de dirigeants politiques dans le développement urbain.

5. Dans le Quintana Roo, le terme de Nouveau Centre de Population Ejidale (NCPE) sert à qualifier les ejidos constitués par l'État mexicain à partir des années 1970 dans le cadre d'une politique régionale de colonisation agraire. Cette distinction permet de différencier ces communautés agraires des ejidos créés au cours d'étapes plus anciennes de la réforme agraire, notamment à partir des années 1930, pour répondre aux demandes foncières des populations locales.
} 
du Tabasco et, plus tard, l'ejido Jacinto Pat constitué en 1987, principalement par des populations mayas, locales ou originaires du Yucatan, ainsi qu'une minorité de paysans en provenance d'autres régions mexicaines. Ces trois ejidos forment la "société agraire » de la localité de Tulum, la grande majorité des ejidatarios résidant dans le bourg où s'étend aujourd'hui la zone urbaine (figure 4 - planche $\mathbf{V}$ ).

La zone habitée de l'ejido Tulum correspond ainsi au bourg historique constitué par les populations mayas autochtones. Celui-ci constitue la partie la plus ancienne de la ville et fut utilisé à différentes époques comme réserve foncière (fundo legal) destinée à accueillir le développement de la zone urbaine. Si les deux autres ejidos ont également été dotés de zones de peuplement situées à plusieurs kilomètres du bourg, celles-ci ne furent jamais réellement habitées, la plupart des ejidatarios ayant élu domicile dans la ville de Tulum.

La formation des ejidos correspond à différents cycles économiques et à différents stades du développement régional, associés à des mouvements migratoires et démographiques. Ces étapes correspondent également à des demandes foncières traduites par une extension de la surface de l'ejido Tulum en 1972 (Marín, à paraître). La structure agraire ejidale s'est ainsi consolidée en l'espace de cinq décennies et réunissait à la fin des années 1980 plus de 250 paysans regroupés au sein d'une même zone de peuplement. Cet ensemble recouvre les trois ejidos sur une surface de près de 42000 hectares. Plus tard, suite au déclin des activités agricoles, au développement urbain et à la valorisation de la terre sur le marché touristique, le nombre des ejidatarios se trouva augmenté (tableau 2). Si ces derniers conservent en théorie une grande partie des réserves territoriales, on assiste en réalité au début d'un processus de fragmentation de la tenure du sol sous l'effet de sa commercialisation, de logiques de transmission intergénérationnelle ou de perte par dépossession. L'une des répercussions les plus visibles de cette fragmentation est la présence croissante de nouveaux acteurs au sein des ejidos, à l'instar des posesionarios et des avecindados. Ces statuts impliquent des rapports à la propriété du sol différents et des droits plus limités que ceux dont jouissent les ejidatarios ${ }^{6}$. Au sein de l'ejido Tulum,

6. Un ejidatario est titulaire des droits agraires sur les terres ejidales attribuées à un groupe de personnes plus ou moins important. Un avecindado on dénombre ainsi six posesionarios et 569 avecindados, ces mêmes chiffres atteignant respectivement 161 et 57 dans le cas de l'ejido Jacinto Pat. L'ejido José Maria Pino Suarez compte quant à lui près de 400 posesionarios détenant des parcelles sur le front de mer. Ce groupe est essentiellement constitué de petits propriétaires d'hôtels ne disposant pas encore de titres reconnus par les autorités agraires et se trouvant actuellement engagés dans des procédures légales auprès des tribunaux agraires ${ }^{7}$.

\section{LES ORIGINES DE L'EJIDO Pino Suarez, de la formation À LA FRAGMENTATION}

L'ejido José Maria Pino Suarez fut initialement constitué comme Nouveau Centre de Population Ejidale (NCPE) dans le cadre d'une politique de colonisation agraire du Quintana Roo menée par le gouvernement fédéral (Mendoza, 2009). Ainsi, un groupe de 47 paysans venus du Tabasco accompagnés de leurs familles reçurent une dotation foncière de 10409 hectares, dont seulement 980 de terres cultivables. Les procédures pour la création de l'ejido furent enclenchées en 1971 et publiées l'année suivante au Journal officiel de la Fédération. En 1973, une résolution présidentielle officialisa la création de l'ejido, rendue effective en $1981^{8}$. Aspect important, la dotation foncière intègre une bande de terres côtières permettant à l'ejido de disposer d'un accès à la mer. Une caractéristique d'autant plus insolite que l'ejido Tulum, dont la création est antérieure, n'a pas eu une telle « chance». Cette période marque le début d'une histoire turbulente dont les principaux protagonistes ne furent pas les paysans du Tabasco, lesquels ont rapidement abandonné leurs terres, mais d'autres groupes dont l'arrivée a permis de perpétuer l'existence légale de l'ejido.

est une personne majeure et de nationalité mexicaine possédant une parcelle ejidale et ayant obtenu ce statut après avoir vécu au moins un an au sein de la communauté agraire. Ce statut est délivré par l'assemblée agraire ou par le tribunal agraire mais n'implique pas les mêmes droits agraires que ceux dont bénéficient les ejidatarios. Enfin, un posesionario est une personne possédant des terres ejidales et un certificat parcellaire conférant un droit d'usage sur une parcelle ou sur des terres communales. Si ce statut est reconnu par l'assemblée ejidale ou par décision de justice, un posesionario ne bénéficie toutefois pas d'un accès de plein droit à la terre.

7. Selon des informations issues du Registre Agraire National (RAN) et de l'enquête de terrain.

8. Selon les données du Registre Agraire National. 


\begin{tabular}{|l|c|c|c|c|c|c|}
\hline Ejido & $\begin{array}{c}\text { Année } \\
\text { de fondation }\end{array}$ & $\begin{array}{c}\text { Nombre } \\
\text { de membres } \\
\text { originels }\end{array}$ & $\begin{array}{c}\text { Nombre } \\
\text { de membres } \\
\text { en 1987 }\end{array}$ & $\begin{array}{c}\text { Nombre } \\
\text { de membres } \\
\text { en 2010 }\end{array}$ & $\begin{array}{c}\text { Nombre } \\
\text { de membres } \\
\text { en 2016 }\end{array}$ & $\begin{array}{c}\text { Surface } \\
\text { (hectares) }\end{array}$ \\
\hline Tulum & 1935 & 35 & 104 & 119 & 162 & 22579 \\
\hline José María Pino Suárez & 1973 & 47 & 47 & 57 & 57 & 10409 \\
\hline Jacinto Pat & 1987 & 105 & 105 & 197 & 206 & 8924 \\
\hline TOTAL & & 187 & 256 & 373 & 425 & 41912 \\
\hline
\end{tabular}

Tableau 2 : Les organisations ejidales à Tulum (1936-2010) (Source: Registre Agraire National) Ejido organizations in Tulum (1936-2010)

Las organizaciones ejidales en Tulum (1936-2010)

Rapidement, la frange côtière de l'ejido fut disputée par deux propriétaires terriens, Antonio González Avilés et Felipe Hernández Collí. Ces derniers revendiquèrent la propriété de 115 hectares de terres, dont 105 revenant supposément au premier. Les autorités agraires estimèrent alors que les terres en question avaient été vendues en concession par le gouvernement fédéral dans le cadre de la colonisation agraire de la région. Comme les parcelles demeurèrent inoccupées, celles-ci restèrent inscrites comme «terrains nationaux ». Et bien que Felipe Hernández Collí disposait d'un titre de possession à perpétuité émis en 1963, ce dernier fut jugé « inefficace » par l'administration agraire. En compensation, les deux particuliers reçurent le statut d'ejidatarios lors de la création de l'ejido. Refusant une telle mesure, ceux-ci continuèrent d'ignorer cette décision et, plus spécifiquement, la délimitation intégrant les terres du front de mer à l'intérieur de l'ejido Pino Suarez.

Paradoxalement, les paysans du Tabasco connurent une grande déception après avoir pris possession des terres attribuées à l'ejido. Leur désillusion résulta notamment de la nature rocailleuse du sol, impropre aux activités agricoles. Malgré cela, ceux-ci procédèrent à la délimitation d'une zone d'habitations située à 18 kilomètres du village de Tulum, à la construction d'abris en feuilles de palmes (palapas) et à l'ouverture d'un chemin menant à la route et à la lagune de Nopalitos. Cependant, l'isolement et l'insalubrité associés à l'absence d'emplois et d'activités productives ont rapidement eu raison de leurs espoirs et précipité l'abandon du lieu. Tandis qu'une partie des paysans fut relocalisée par l'administration agraire dans un autre ejido situé dans les environs de Chetumal, près de la frontière bélizienne, une autre partie des ejidatarios choisirent de rentrer dans leur village d'origine dans le Tabasco. Simultanément, le lieu commença à accueillir des personnes venues d'autres régions mexicaines. Ce remplacement progressif commença avec un groupe d'une dizaine de familles originaires de l'état de Jalisco ayant appris l'existence d'un «ejido abandonné ». De la même manière, des familles commencèrent à arriver de la région de Guanajuato, ainsi que des travailleurs venus de Cancun et des mayas ayant émigré depuis le Yucatan. Arrivèrent également des artisans et quelques aventuriers installés à Tulum. En général, l'arrivée de nouveaux occupants est alors d'autant mieux acceptée qu'elle coïncide avec l'intérêt des locaux d'accroitre le peuplement afin de pérenniser l'existence légale de l'ejido.

Un an après la création de l'ejido, Antonio González Avilés - l'un des deux propriétaires revendiquant la propriété des terrains côtiers - obtint illégalement l'inscription à son nom d'une parcelle de 49 hectares au Registre Public de la Propriété et du Commerce de l'État du Quintana Roo. Cet enregistrement est effectué avec l'appui de la directrice des Nouveaux Centres de Population Ejidale sur présentation d'un titre de propriété émis en 1948 et n'ayant pas été identifié par l'administration agraire lors des études préalables à la création de l'ejido. La parcelle est ensuite fractionnée en treize lots par le biais d'une société fiduciaire puis revendue à deux entrepreneurs originaires de la région de Monterrey, dans le nord du Mexique, Esteban Eschiavon Magaña et José María Garza Ponce. Ces derniers procèdent à leur tour à la fragmentation de la propriété et à sa revente partielle à d'autres investisseurs. L'ensemble en question correspond au site connu sous le nom de Punta Piedra et représente une frange littorale d'environ douze kilomètres de long. 
Les particuliers revendiquant la propriété des terres de Punta Piedra ont alors disposé de nombreux panneaux de part et d'autre de la route littorale pour signaler que les terres en question constituaient une propriété privée. Si cette mesure visait probablement à dissuader d'éventuels envahisseurs, les entretiens effectués auprès des ejidatarios montrent qu'à cette époque une telle signalisation ne risquait pas d'intimider qui que ce soit. En réalité, les conditions d'existence précaires, les moustiques ou encore l'absence de services, de commerces et d'emplois furent des facteurs bien plus déterminants ayant contribué à décourager les différentes tentatives de peuplement. Pendant les années suivantes, certains habitants quittèrent la localité tandis que d'autres arrivèrent mais la population du lieu n'augmenta pas. L'abandon relatif de l'ejido et l'instabilité de sa population ont ainsi permis aux entrepreneurs de faire pression sur les autorités agraires pour que celles-ci attestent de l'absence des bénéficiaires originels de la dotation, espérant ainsi remettre en cause l'existence légale de l'ejido ${ }^{9}$. Pour autant, les populations résidentes du lieu jouèrent le rôle initialement attribué aux ejidatarios et, avec l'appui des mêmes autorités, entreprirent des procédures visant au contraire la régularisation de l'ejido. Cette démarche s'accéléra lorsque la population commença à prendre conscience de la valeur potentielle des terres et de leur importance dans le contexte du développement touristique.

Ces différentes pressions aboutirent en avril 1981 à la délimitation définitive de l'ejido par le ministère de la réforme agraire. La délimitation fut effectuée lors d'une visite d'à peine cinq heures au cours de laquelle les acteurs présents sont censés avoir parcouru l'ensemble des terres pour procéder à des mesures. Un tel procédé implique que ceux-ci aient pu parcourir 48 kilomètres de jungle et de mangrove dans des conditions climatiques particulièrement difficiles du fait de la chaleur. Il est alors évident pour les ejidatarios que le polygone ejidal atteignait la côte, bien que cet aspect n'apparaisse pas explicitement dans le document qu'ils eurent à signer

9. Selon les informations du Registre Agraire National, le délégué du ministère de la réforme agraire adressa en janvier 1981 un courrier au président de la municipalité de Cozumel en lui demandant d'attester du fait que les bénéficiaires de l'ejido José Pino Suarez ne s'étaient pas présentés afin d'occuper les terres reçues dans le cadre de la dotation ejidale. Le 2 février de la même année, le président municipal de Cozumel certifia ainsi que les personnes ayant sollicité les terres concernées n'en prirent jamais possession. ce jour-là. À ce moment, seuls 19 des 47 paysans du Tabasco ayant bénéficié de la dotation agraire signèrent ou apposèrent leur empreinte digitale sur le procès-verbal. Plus tard, lors de l'émission de plusieurs documents officiels, ces derniers s'aperçurent que, aussi incroyable que cela puisse paraître, l'ejido José Maria Pino Suarez n'atteignait pas le front de mer. La création de l'ejido fut rendue officielle par une résolution présidentielle en 1982.

Quelques années plus tard, en 1989 et en 1992, des démarches furent engagées pour réduire le nombre d'ejidatarios ${ }^{10}$. En 1989, l'ejido ne comptait en effet plus aucun des paysans tabasqueños initialement concernés par la dotation agraire. Face au risque de voir disparaître l'ejido - la loi mexicaine exigeant qu'au moins cinq membres originels de l'ejido continuent d'occuper la terre pour assurer sa pérennité juridique - les habitants nommèrent un comité chargé de se rendre dans le Tabasco à la recherche des paysans fondateurs de l'ejido. Après un long périple, les intéressés parvinrent à retrouver la trace de cinq d'entre eux et à les convaincre de revenir à Tulum pour reprendre possession des terres. En septembre 1989, l'administration agraire émis finalement un document certifiant les droits agraires des 47 membres de l'ejido José Maria Pino Suarez. Dans la nouvelle liste des bénéficiaires de la dotation ejidale, seuls cinq correspondent donc aux membres originels de l'ejido, le reste de la communauté agraire étant composé de personnes établis dans l'ejido au fil des années. En d'autres termes, les terres furent distribuées à un groupe très différent de celui initialement constitué, bien qu'étant situé au même endroit et désigné par le même nom.

\section{L'INTENSIFICATION ET LA COMPLEXIFICATION DES LUTTES POUR LE TERRITOIRE}

Le conflit pour le contrôle des plages de Tulum débuta en 1992 lorsque l'ejido initia un certain nombre de procédures officielles dans le cadre du Programme de Certification des Droits Ejidaux (PROCEDE) mis en œuvre par le gouvernement fédéral. Ce dispositif destiné à faciliter la titrisation

10. D’après le Registre Agraire National. Cette procédure dite de «depuración ejidal » consiste à annuler les droits agraires de certains ejidatarios, notamment en cas de non-occupation des parcelles reçues lors de la dotation agraire. 
des terres ejidales, à leur fournir une sécurité juridique et à permettre leur commercialisation s'inscrit dans un processus plus global de libéralisation du marché foncier à travers la réforme de l'article 27 de la Constitution mexicaine adoptée la même année ${ }^{11}$. Cependant, les ejidatarios se virent refuser l'incorporation au PROCEDE dans la mesure où l'intégration de la plage dans l'ejido fut jugée irrecevable par l'administration agraire au regard de la délimitation effectuée en 1981. Il devint alors évident que l'ejido avait été dépossédé de sa partie littorale qui n’apparaissait pas dans les plans officiels. La décision provoqua la colère des ejidatarios, convaincus d'avoir été trahis par les autorités, et déclencha une série des heurts violents. Les ejidatarios décidèrent aussitôt d'occuper les terrains côtiers et de s'engager dans une "lutte à mort » (lucha a muerte $)^{12}$ contre ce qui leur apparaissait comme un acte frauduleux. Si le gouvernement leur reconnaissait bien la possession de 10409 hectares comme cela était initialement convenu, celui-ci les avait également privés de l'espace doté de la plus grande valeur.

Simultanément, et avec l'espoir d'obtenir à terme la titrisation des terres, les ejidatarios procédèrent au fractionnement de la quasi-totalité de l'ejido et se répartirent les parcelles en préservant une certaine équité du point de vue de la valeur des terres et de leur usage potentiel. Cette démarche marqua le début d'un processus de commercialisation des parcelles et de nombreux particuliers firent l'acquisition d'un terrain sur la plage ${ }^{13}$. Quelques ejidatarios ont rapidement dilapidé le patrimoine ainsi constitué tandis que d'autres, bénéficiant d'une meilleure connaissance du marché foncier et des procédures administratives, optèrent pour la prudence et vendirent la terre à un meilleur prix. Certains d'entre eux investirent dans des commerces, parfois en s'associant avec de petits propriétaires d'hôtels. D'autres ont simplement conservé la plus grande partie de leurs parcelles, dont la valeur se compte aujourd'hui en milliers de dollars. Ce phénomène contribua à engendrer une mosaïque de petits propriétaires - près de 400 - disposant de titres émis par les autorités de l'ejido. Si de tels docu-

11. Cette réforme constitue notamment un préalable à la mise en œuvre de l'ALENA, accord de libre-échange entrant en vigueur deux ans plus tard. 12. Propos recueillis dans le cadre d'entretiens avec des membres de l'ejido.

13. Certaines transactions sont ainsi effectuées en dehors des cadres légaux. Dans d'autres cas, prévus par la législation agraire, la transaction ne porte pas sur la propriété de la parcelle mais sur la cession des droits agraires à un tiers en échange d'une somme d'argent. ments leur garantissent la possession de la terre, ils leur procurent en revanche une faible sécurité sur le plan légal.

Dans ce contexte, les descendants des familles du nord du pays ayant acheté les mêmes terres à Antonio González Avilés refirent leur apparition en 1993, non sans avoir eux-mêmes fractionné leurs parcelles respectives pour les transmettre à des proches ou à des associés. Ceux-ci tentèrent d'expulser les ejidatarios, notamment à travers des recours en justice pour occupation et vente illégale de terre. En 1995, ils déposèrent officiellement plainte contre les ejidatarios avec l'appui du gouverneur du Quintana Roo, Mario Villanueva, afin d'obtenir leur expulsion. Parallèlement, une vague de répression policière fut mise en œuvre contre les leaders agraires locaux et les ejidatarios - hommes et femmes - qui subirent une série de passages à tabac et d'arrestations. Cette stratégie d'intimidation vise alors à les contraindre à renoncer à la propriété des terrains côtiers ${ }^{14}$. Si certains leaders locaux ont passé plus d'un an en prison, la plupart des habitants continua cependant d'occuper les lieux.

Aujourd'hui encore, les ejidatarios continuent de disputer envers et contre tous l'espace concerné à travers des moyens légaux et par différents subterfuges, mais aussi par la force. Sur le plan juridique, les membres de l'ejido ont entrepris un recours visant l'annulation du titre de propriété du prétendu propriétaire de la frange côtière ainsi que des documents postérieurs issus de la fragmentation de la propriété, mettant notamment en cause l'authenticité du document originel. Dès 1994, les plaignants saisissent la Cour Suprême de Justice de la Nation - la plus haute instance judiciaire mexicaine - en dénonçant la résolution présidentielle de 1981 privant l'ejido de sa partie côtière. L'argument est jugé d'autant plus central que des documents plus anciens, établis au cours du processus de dotation foncière, précisent que l'ejido atteint le rivage. Depuis 1997, ils eurent également recours aux tribunaux agraires et s'engagèrent dans un litige long et fastidieux. En 2012, alors que les démarches engagées connaissaient un certain nombre de progrès importants, l'avocat de l'ejido et de l'association de petits entrepreneurs touristiques de sa partie litto-

14. D’après les entretiens réalisés avec des membres fondateurs de l'ejido. 
rale fut assassiné sans que les responsables du crime ne soient inquiétés.

Dans cette nouvelle phase du conflit, les investisseurs du nord du Mexique revinrent à la charge, non seulement contre les ejidatarios mais aussi contre les propriétaires d'hôtels accusés d'occupation illégale. Les pressions exercées se traduisirent par des mesures légales ou extralégales et des procès civils menant à une série de condamnations et d'opérations de police pour récupérer les terrains disputés, avec l'aval des autorités locales. En 2009, les investisseurs du nord obtinrent la confiscation d'un premier hôtel et la mise en échec d'autres petits propriétaires, suscitant la résistance des locaux qui réclamèrent la protection du gouvernement du Quintana Roo. Malgré cela, les investisseurs reprirent le contrôle de 26 hôtels, de 13 cabanes et d'une cinquantaine de terrains situés sur le front de mer entre 2011 et $2016^{15}$. En juillet 2016, après une vague d'expulsions ciblant les hôtels locaux, le conflit attira l'attention de médias internationaux (CNN, New York Times, Forbes, El Pais) rendant compte d'abus de la part des autorités locales et d'atteintes contre un grand nombre de propriétaires étrangers et de touristes. Laffaire devint un scandale national, notamment après la publication de reportages ${ }^{16}$ exposant la manière dont ces opérations témoignaient d'une stratégie par laquelle un réseau criminel dirigé par l'ex-gouverneur du Quintana Roo, Roberto Borge, et impliquant des politiciens, des entrepreneurs ainsi que des juges et des fonctionnaires gouvernementaux, se livrait au pillage du patrimoine public, à la fraude et à la spoliation de terres publiques ou privées. Entre d'autres termes, un mécanisme de corruption et de spoliation situé au cœur du système politique et institutionnel.

\section{QUAND LA SPÉCULATION FONCIÈRE ATTISE LES CONFLITS LOCAUX}

Au-delà des éléments évoqués jusqu'ici, la complexité de la situation traitée se trouve accentuée par le phénomène de spéculation immobilière, mais aussi par l'implication d'un autre groupe d'acteurs participant de ce système de mafia agraire. En effet,

15. I. Maribel S. Meza, «Los piratas de Borge. El saqueo de bienes institucionalizado en Quintana Roo », journal Expansión, 6 juillet 2016.

16. On retiendra en particulier celui réalisé par la journaliste mexicaine Lydia Cacho (2015). l'ex-banquier Roberto Hernandez, doté d'une grande influence au niveau national et s'étant approprié d'importantes surfaces de terres dans la péninsule du Yucatan au cours des dernières années, a utilisé son pouvoir afin d'accaparer les terres de l'ejido. Ceci avec l'appui d'un groupe d'opérateurs notoires, d'intermédiaires, d'associés et de prête-noms habitués à faire la une de la presse locale.

Initialement, la relation entre le banquier et l'ejido remonte à 1992 et fut établie lorsque la communauté agraire sollicita une aide juridique pour la délimitation et la titrisation de ses terres dans le cadre du programme PROCEDE. Dans le même temps, et à travers différents opérateurs, celui-ci se chargea de convaincre les ejidatarios de vendre leurs droits agraires, si bien qu'en 2005 le groupe en question avait acquis les droits de 33 ejidatarios soit plus de la moitié des membres de la communauté agraire. Ce procédé s'opéra par différents moyens et, plus spécifiquement, par le recours à des associés et des proches utilisés comme prêtenoms ${ }^{17}$. C'est dans ce contexte qu'une délimitation et une cartographie de l'ejido fut approuvée en 2006 par une assemblée agraire aux cours de laquelle les ejidatarios se répartirent les différentes fractions de plages et, plus largement, l'ensemble de l'ejido. Avec la reconnaissance de cette démarche par le Registre Agraire National en 2009, le groupe de spéculateurs mené par Roberto Hernandez obtint légalement le statut d'ejidata$\operatorname{rios}^{18}$. Grâce au contrôle de ces nouveaux ejidatarios devenus majoritaires, ajouté à la complicité de certains commissaires ejidaux, le banquier parvint progressivement à contrôler les assemblées ejidales et les décisions les plus importantes de la communauté agraire. Cependant, la résistance de certains ejidatarios a été importante et a même conduit, à certains moments, à la coexistence de deux commissaires ejidaux distincts, reconnus par leurs soutiens respectifs au sein de la communauté. Malgré tout, les membres de l'ejido demeurèrent unis par leur intérêt commun de conserver la possession des terrains côtiers et de faire reconnaître leurs droits par les tribunaux agraires.

17. Ces faits ont pu être établis à partir des entretiens effectués avec les membres fondateurs de l'ejido parmi lesquels d'anciens commissaires ejidaux.

18. Informations issues du Registre Agraire National (RAN). 


\section{Conclusion et RÉfLEXIONS FINALES}

Le cas de l'ejido Pino Suarez illustre trois problèmes fondamentaux : l'impossibilité de définir légalement, et de manière transparente et précise, les limites du territoire ejidal ; l'impossibilité de déterminer l'identité des propriétaires légitimes de sa frange côtière et, finalement, l'impossibilité de faire valoir l'état de droit en ce qui concerne la propriété de la terre. Plus de quarante ans après la création de l'ejido, les autorités demeurent incapables de répondre à ces enjeux et ont montré leur indolence, leur partialité et leur incompétence en la matière. L'une des principales raisons d'une telle situation réside précisément dans le fait que les institutions publiques se trouvent immergées dans un enchevêtrement d'intérêts et de pratiques de corruption liés au grand capital cherchant à s'approprier l'espace, dans un contexte de spéculation immobilière et de projets touristiques de grande ampleur. Ainsi, il s'agit d'une lutte acharnée dans laquelle l'ensemble des protagonistes en appellent à la loi et aux voies institutionnelles tout en recourant à tous les moyens possibles parmi lesquels la fraude et la violence. Pour autant, cette lutte reste marquée par le poids des asymétries sociales et des rapports de domination dans la mesure où des grands capitaux liés à des mafias agraires semblent s'imposer au détriment des ejidatarios et des petits entrepreneurs locaux. Parmi ces derniers, certains ont pu résister malgré d'intenses pressions dans un climat d'intimidations et d'incertitudes, au prix de longs et couteux procès motivés par le rêve d'un avenir prospère.

Pour autant, l'issue d'un tel conflit demeure ouverte. Les controverses publiques suscitées par ce cas de spoliation, les nombreuses enquêtes journalistiques sur les délits commis par l'ex-gouverneur du Quintana Roo Roberto Borje Angulo et ses complices ainsi que l'élection d'un nouveau gouverneur en 2016 ont débouché sur plusieurs procédures judiciaires amenant à l'arrestation du gouverneur sortant en 2017 et à son incarcération. Si ce retournement de situation n'a pas conduit à l'appréhension de l'ensemble des acteurs impliqués dans ces logiques frauduleuses, le nouveau climat politique dans l'état du Quintana Roo a néanmoins permis de mettre un terme aux actions menées contre les ejidatarios et des petits hôteliers de Tulum, dont une partie a déjà pu bénéficier d'une restitution de leurs biens. 


\section{Annexe 1 : Chronogramme récapitulant les différentes étapes du conflit foncier}

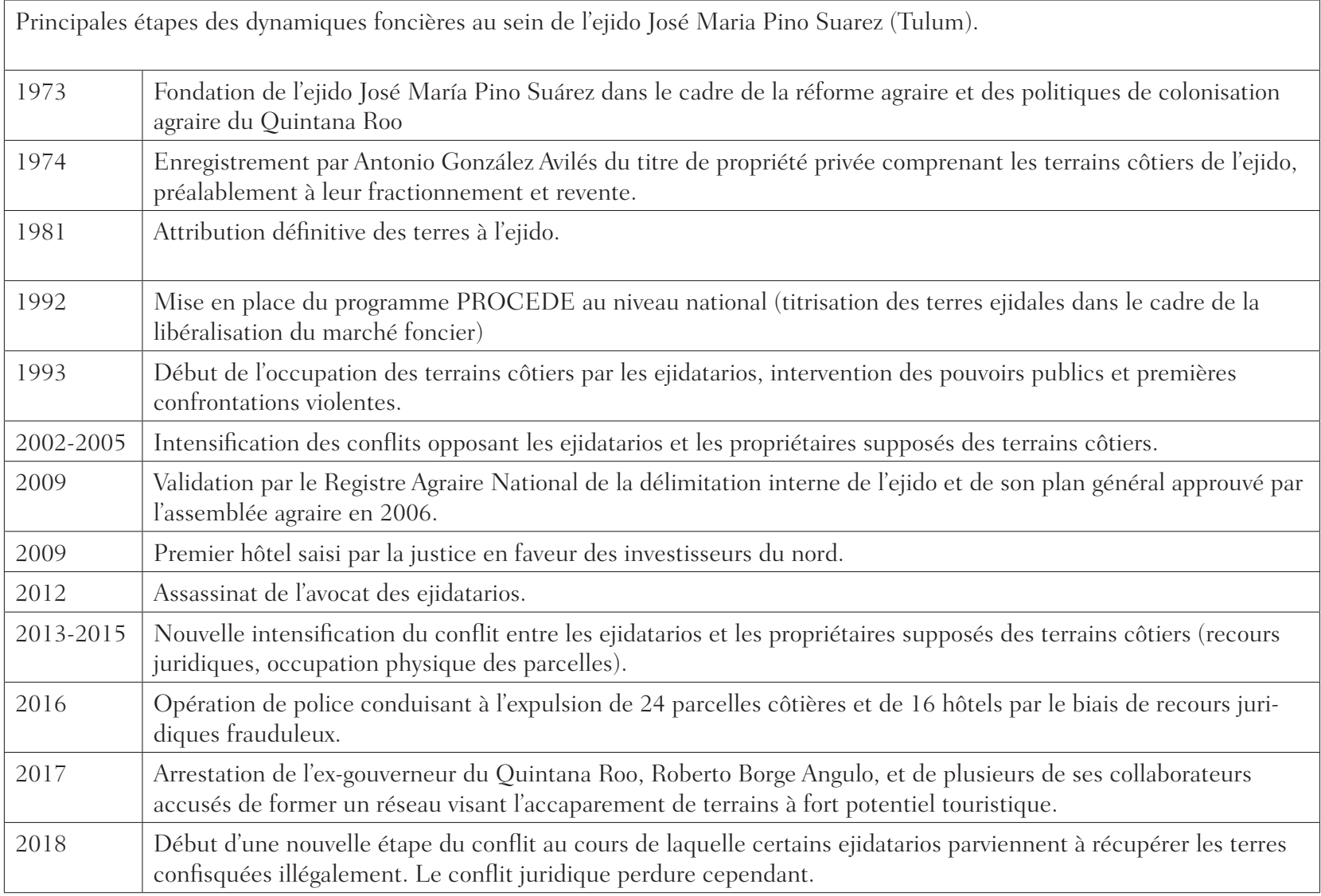

\section{Bibliographie}

Expansión, 2017. « Hoteles despojados en Tulum son devueltos a sus propietarios », Expansión, en alianza con CNN, 11 de octubre, en ligne. [https://expansion.mx/nacional/2017/10/11/hoteles-despojados-en-tulum-son-devueltos-a-sus-propietarios].

Cacho Lydia, 2015. «Tulum: tierra de ambiciones », en Newsweek, septiembre 06, [http://nwnoticias.com/\#!/noticias/ tulum-tierra-de-ambiciones], consultado el 07 de octubre de 2015.

Harvey David, 2003. El nuevo imperialismo, Madrid, ediciones Akal, $175 \mathrm{p}$.

Ibarra Maribel, Meza Silber, 2016. « Los piratas de Borge. El saqueo de bienes institucionalizado en Quintana Roo », en Expansión/ Mexicanos contra la corrupción y la impunidad, julio 06, [http://expansion.mx/nacional/2016/07/06/ los-piratas-de-borge-el-saqueo-de-bienes-institucionalizadoen-quintana-roo].

INEGI, 2015. Encuesta Intercensal, México, Instituto Nacional de Estadística y Geografía,[http://www.beta.inegi.org.mx/ proyectos/enchogares/especiales/intercensal/].
JuÁrez Ana María, 2002. «Ecological degradation, global tourism, and inequality: Maya interpretations of the changing environment in Quintana Roo, Mexico », Human Organization, Vol. 61, Núm. 2, p. 113-124.

López SAntillán Ángeles, 2010. Metamorfosis del paraíso. La producción de Holbox como lugar turístico del Caribe mexicano, Tesis de doctorado, Zamora, México. El Colegio de Michoacán, 533 p.

López Santillán Ángeles y Marín Guardado Gustavo, 2010. « Turismo, capitalismo y producción de lo exótico: Una perspectiva crítica para el estudio de la mercantilización del espacio y la cultura ", Relaciones. Estudios de Historia y Sociedad, Vol. XXXI, Núm. 123, verano, p. 219-258.

Marín Guardado Gustavo, 2010. «Turismo, globalización y mercantilización del espacio y la cultura en la Riviera Maya: un acercamiento a tres escenarios », en Ricardo López Santillán (ed.), Etnia, lengua y territorio. El sureste frente a la globalización, México, UNAM, p. 17-55.

Marín Guardado Gustavo (coord.) 2015. Sin tierras no hay paraíso. Turismo, sociedades agrarias y apropiación territorial en México, Colección Pasos Edita No. 15, El Sauzal, 
Tenerife, España, PASOS. Revista de Turismo y Patrimonio Cultural, 2015, $310 \mathrm{p}$

Marín Guardado Gustavo (à paraître). Turismo y mercantilización del espacio y la cultura en Tulum, Quintana Roo, México, CIESAS.

Mendoza Ramírez Martha Patricia, 2009. Políticas de colonización en Quintana Roo 1958-1980, Tesis de doctorado en Humanidades, Línea historia, División de Ciencias y Humanidades, Universidad Autónoma Metropolitana, Unidad Iztapalapa, México, 267 p.

Registro Agrario Nacional (RAN), 2017. Padrón Histórico de Núcleos Agrarios, Registro Agrario Nacional, disponible en [http://phina.ran.gob.mx/], consultado el 04 de junio de 2017.

Registro Agrario Nacional (RAN), s/f. Carpeta Básica de Dotación del Nuevo Centro de Población Ejidal José María Pino Suárez, Registro Agrario Nacional, delegación Quintana Roo, Chetumal, México.

Registro Agrario Nacional, s/f. Carpeta Básica de Dotación del Ejido Jacinto Pat, Registro Agrario Nacional, delegación Quintana Roo, Chetumal, México.

Registro Agrario Nacional, s/f. Carpeta Básica de Dotación del Ejido Tulum, Registro Agrario Nacional delegación Quintana Roo, Chetumal, México.
SAlmerón CASTRo Fernando, 1998. Intermediarios del progreso: política y crecimiento económico en Aguascalientes, México, Centro de Investigaciones y Estudios Superiores en Antropología Social-Instituto Cultural de Aguascalientes. $313 \mathrm{p}$

SECTUR, 2017. Registra turismo cifras históricas en 2016: 35 millones de visitantes y 19,571 MDD en divisas, 13 de febrero, Secretaría de Turismo (Sectur), Prensa, [https:// www.gob.mx/sectur/prensa/registra-turismo-cifras-historicas-en-2016-35-millones-de-visitantes-y-19-571-mdd-endivisas].

SEDETUR, 2016. «Indicadores turísticos enero a diciembre de 2016 », Secretaría de Turismo de Gobierno del Estado de Quintana Roo, consultado el 04 de agosto de 2017 [http:// sedetur.qroo.gob.mx/estadisticas/indicadores/2017/Indicadores\%20Tur\%20-\%20Enero\%20-\%20Diciembre\%202016. pdf].

Tribunal Unitario Agrario, 2006. Expediente TUA 44-131/2006, Distrito 44, Chetumal, Quintana Roo, México.

VARILlas Adriana, 2017. «Restituyen tres hoteles en Tulum a sus dueños originales », El Universal, 10 de octubre, [http:// www.eluniversal.com.mx/estados/restituyen-tres-hoteles-entulum-sus-duenos-originales]. 